\title{
Prevalence and correlates of tobacco use amongst junior collegiates in twin cities of western Nepal: A cross-sectional, questionnaire-based survey Chandrashekhar T Sreeramareddy*1, PV Kishore ${ }^{2}$, Jagadish Paudel ${ }^{3}$ and Ritesh G Menezes ${ }^{4}$
}

Address: ${ }^{1}$ Department of Community Medicine, Manipal Teaching Hospital, Manipal College of Medical Sciences, Pokhara, Nepal, ${ }^{2}$ Department of Internal Medicine, Manipal Teaching Hospital, Manipal College of Medical Sciences, Pokhara, Nepal, ${ }^{3}$ Department of Sociology, Prithvi Narayan Campus, Pokhara, Nepal and ${ }^{4}$ Department of Forensic Medicine and Toxicology, Kasturba Medical College, Mangalore, India

Email: Chandrashekhar T Sreeramareddy* - chandrashekharats@yahoo.com; PV Kishore - pandu_vki@yahoo.com; Jagadish Paudel - paudeljg@hotmail.com; Ritesh G Menezes - mangalore971@yahoo.co.in

* Corresponding author

Published: 26 March 2008

BMC Public Health 2008, 8:97 doi: I0.I |86/147|-2458-8-97
Received: 18 July 2007

Accepted: 26 March 2008

This article is available from: http://www.biomedcentral.com/I47I-2458/8/97

(c) 2008 Sreeramareddy et al; licensee BioMed Central Ltd.

This is an Open Access article distributed under the terms of the Creative Commons Attribution License (http://creativecommons.org/licenses/by/2.0), which permits unrestricted use, distribution, and reproduction in any medium, provided the original work is properly cited.

\begin{abstract}
Background: College students are vulnerable to tobacco addiction. Tobacco industries often target college students for marketing. Studies about prevalence of tobacco use and its correlates among college students in Nepal are lacking.

Methods: A cross-sectional survey was carried out in two cities of western Nepal during January-March, 2007. A pre-tested, anonymous, self-administered questionnaire (in Nepali) adapted from Global Youth Tobacco Survey (GYTS) and a World Bank study was administered to a representative sample of 1600 students selected from 13 junior colleges by two-stage stratified random sampling.

Results: Overall prevalence of 'ever users' of tobacco products was $13.9 \%$. Prevalence among boys and girls was $20.5 \%$ and $2.9 \%$ respectively. Prevalence of 'current users' was $10.2 \%$ (cigarette smoking: $9.4 \%$, smokeless products: $6.5 \%$, and both forms: $5.7 \%$ ). Median age at initiation of cigarette smoking and chewable tobacco was 16 and I 5 years respectively. Among the current cigarette smokers, 58.7\% (88/I50) were smoking at least one cigarette per day. Most (67.8\%) 'Current users' purchased tobacco products by themselves from stores or got them from friends. Most of them (66.7\%) smoked in tea stalls or restaurants followed by other public places (I3.2\%). The average daily expenditure was 20 Nepalese rupees $(\sim 0.3$ USD) and most (59\%) students reported of having adequate money to buy tobacco products. Majority $(82 \%)$ of the students were exposed to tobacco advertisements through magazines/newspapers, and advertising hoardings during a period of 30 days prior to survey. The correlates of tobacco use were: age, gender, household asset score and knowledge about health risks, family members, teachers and friends using tobacco products, and purchasing tobacco products for family members.

Conclusion: School/college-based interventions like counseling to promote cessation among current users and tobacco education to prevent initiation are necessary. Enforcement of legislations to decrease availability, accessibility and affordability of tobacco products and policies to change social norms of tobacco use among parents and teachers are necessary to curb the tobacco use among college students.
\end{abstract}




\section{Background}

Tobacco use is one of the leading preventable causes of premature death, disease and disability around the world [1]. An estimated 4.9 million deaths occurring annually can be attributed to tobacco use. This figure is expected to rise to about 10 million by the year 2020, if the current epidemic continues and more than $70 \%$ of these deaths are expected to occur in developing countries [2]. In Nepal, chronic non-communicable diseases account for $42 \%$ of all the deaths [3]. This rate may be due to a high prevalence of current daily tobacco users among men and women aged 18 years and above which was $48.5 \%$ and $24 \%$ respectively [4]. In the mountain region, the reported prevalence of females smoking was $71.6 \%$ [5]. Various studies have reported prevalence ranging from $20 \%$ to $72 \%$ in different populations of Nepal [5-8]. A study from eastern Terai reported a prevalence of $12.9 \%$ and $14.1 \%$ respectively for cigarette smoking and smokeless tobacco use among women [8]. The Global Tobacco Surveillance System (GTSS) collaborative group has reported that tobacco use (both cigarette and smokeless tobacco) among those aged 13-15 years is high and has emphasized that interventions are necessary for the prevention of initiation and to promote cessation of tobacco among current users [9]. The GYTS, Nepal (2001) has reported a prevalence of $16.3 \%$ for any form of tobacco use among the high schools students of central region [10].

Adolescents and young adults of colleges are often targeted by the tobacco industry for marketing. Moreover, college age is a transition period and students are vulnerable to tobacco addiction. Like high schools even colleges can be targeted for tobacco control interventions. A study from India reported that the prevalence of tobacco use is high and popular perceptions exist about tobacco products among students [11]. Studies about tobacco use and its correlates among college students in Nepal are lacking. Studies about awareness/knowledge of the risks of smoking are also lacking. Moreover, the previous studies including GYTS, Nepal [10] have not explored the factors determining tobacco use behavior among college students. A World Bank study from Indonesia identified various predisposing, enabling and reinforcing factors which determined tobacco use among the Indonesian youth in high schools [12]. These factors may be specific to culture, traditions and other characteristics of a country. Identification of such factors may be potentially useful to formulate policy interventions needed towards behavior change communication for prevention and control of tobacco use among college students. Therefore, we carried out the present study with the following objectives:

1. to estimate the prevalence of tobacco use, and

2. to assess the correlates of tobacco use.

\section{Methods \\ Study area}

Nepal is a poor developing country in the South-east Asia region of the World Health Organization (WHO). In 2002, Nepal was ranked $140^{\text {th }}$ among 174 countries in the Human Development Index and with a Gross Domestic Product 0.44 [13]. It has a total land area of 147,000 square kilometers. According to the 2001 census, the total population of Nepal was 23.15 million (male: 11.56 million and female: 11.58 million) and the sex-ratio was 998 males for 1000 females. As a consequence of a high growth rate, majority of population of the country is fairly young. About $39.3 \%$ of the total population is in the age group $0-14$ years and only $6.5 \%$ are above 60 years of age. Eighty-one per cent of the economically active population is employed in the agricultural sector. Nepal is a proclaimed Hindu Kingdom. Eighty percent of the population is Hindus, followed by Buddhists (10.7\%) and Muslims (4.2\%). Only $48.1 \%$ of males above 14 years of age have minimum high school level education while for females the percentage was 27.2. Kaski district is one of the 14 districts in western development region of Nepal. The district has a land area of 2017 square kilometers and a population of 380527. Kaski district has 43 village development committees, Pokhara sub metropolitan city and Leknath municipality whose populations are 156312 and 41369 respectively [14].

\section{Conceptual frame work of the study}

We followed the same conceptual frame work as the World Bank study on tobacco economics carried out in Indonesia (2003) [12]. This study was based on Green's (1991) PRECEDE (Predisposing, reinforcing and enabling factors in communication and educational diagnostic evaluation) model [15]. According to the PRECEDE model, three important groups of factors play an important role in changing the behavior and the environment. These are:

\section{1) Predisposing factors, \\ 2) Enabling factors, and \\ 3) Reinforcing factors.}

Based on these factors educational and organizational strategies can be implemented for behavior and environment change [15].

\section{Study design}

A cross-sectional, anonymous self-administered questionnaire survey was carried out.

\section{Questionnaire}

The English versions of the questionnaires used for the Global Youth Tobacco Survey, 2001 [16] and World Bank 
study on tobacco economics, Indonesia, 2003 [12] were reviewed. The authors were contacted and permission was obtained to use the questionnaire for this survey. The questionnaire was adapted and modified from those mentioned above to suit the cultural sensitivity of Nepal. The questionnaire thus prepared in English was translated into local vernacular language, Nepali by JP (Jagadish Paudel). The Nepalese version of questionnaire was pretested among 50 students of a publicly-funded junior college. The necessary modifications were made after pretesting. The final version of the questionnaire was backtranslated into English by two third year undergraduate Nepalese medical students. The questionnaire contained items on demographic data, age at initiation, frequency and type of tobacco products used. The questionnaire also contained questions on knowledge about the health risks of tobacco use, beliefs and attitudes towards tobacco use, tobacco use habits among friends, family members and teachers, exposure to tobacco advertisements and efforts towards cessation of tobacco use. The questions about tobacco-related school curriculum and exposure to environmental tobacco smoke from GYTS questionnaire were not included.

\section{Definitions of the variables \\ Ever users}

Ever smoker or chewer was defined as one who had not smoked/chewed tobacco in the past 30 days preceding the survey but had tried in the past (even once or twice).

\section{Current tobacco user}

Current smoker or chewer was defined as those who had smoked/chewed tobacco product on one or more days in the preceding month of the survey.

\section{Household asset score and categories}

We used household asset score as a proxy to the economic status of the students. Eight items: radio, bicycle, television, fridge, motor-bike, washing machine, computer and car present at their homes were indicated in the demography section of the questionnaire. Each item was given a score ranging from 5 to 40 with a maximum total score of 180 if all the seven items were present (For example, radio was given least score of 5 and car was given the highest score of 40). For each student the total score was calculated and divided into one of the three categories i.e. low (0-60) middle (61-120) and high (121-180).

\section{Knowledge score}

Ten questions on knowledge about the harmful effects of smoking were included in the questionnaire. For each question correct response was given a score of one and incorrect or 'don't know' was scored as zero. The sum giving a minimum score of zero and a maximum of 10 was used as a continuous variable.

\section{Sample size determination and sampling method}

The GYTS, Nepal (2001) reported that overall 16.3\% of the students belonging to $8^{\text {th }}-10^{\text {th }}$ grade had ever used tobacco product in any form [10]. Among the junior college students ( $11^{\text {th }}$ and $12^{\text {th }}$ grades) we expected a minimum prevalence of tobacco use to be $20 \%$. The required minimum sample size to estimate the prevalence was calculated for $95 \%$ confidence limits and an allowable error of $10 \%$ was 1600 .

A two stage stratified random sampling method was used to select a representative sample of students. There were seven junior colleges in Leknath municipality and 30 in Pokhara Sub Metropolitan City. A list of all the junior colleges and their enrollment sizes was obtained from the district secondary education board. At the first stage, five publicly-funded colleges and eight private colleges were randomly selected by stratification according to type of college (private/public) from both the cities. The college enrollment size varied from 25 in private colleges to approximately 6,000 in publicly-funded colleges. Publicly-funded colleges had the largest enrollment size. In the second stage, in colleges with an enrollment size of 100 or less, all the students were included. Two or more classes were randomly selected by lottery method from those colleges with an enrollment size of more than 100 . The largest of all the colleges was Prithvi Narayan Campus affiliated to Tribhuvan University. The college had about approximately 6000 students in different streams of study. Therefore, five classes belonging to different streams of study were selected for survey.

\section{Data Collection}

A written permission was obtained from the office of the district secondary education board. The research ethics committee of the Manipal College of Medical Sciences approved this study. The survey was carried out between January and April, 2007. The researchers (CTS and JP) were involved in administering the survey instrument (questionnaire) to the students. The principals/institutional chiefs of the selected college were personally contacted by the researchers. The objective and nature of the study was explained and a verbal consent was sought to carry out the survey in the college. All the students present at the time of our visit were included for the survey. The students of the selected class were assembled in their class room. The purpose of the survey was explained and assurance about the confidentiality of the information provided was given to the students. After such briefing, they were invited to participate in the survey. Informed consent of the students was sought and the students were informed that they were free to opt not to participate in the survey. Absence of the school personnel/teachers in the classrooms was ensured to encourage the students respond without reporting bias. The printed copies of the 
anonymous, self-administered questionnaires in Nepali were distributed to the students. The completed questionnaires were collected by the researchers.

\section{Data Analysis}

Data were coded and entered into Microsoft excel package and extracted into SPSS (Statistical Package for Social Sciences) version 10 for windows. Rates, percentages and descriptive statistics were calculated. The 'ever use' of tobacco products in any form was used as dependant variable. Demographic characteristics, predisposing, enabling and reinforcing factors were treated as independent variables. Multivariate logistic regression analyses were carried out by 'enter' method. All the groups of variables were entered into the regression model. Odds ratios (OR), $95 \%$ confidence intervals (95\% CI) and p-values were calculated for each independent variable.

\section{Results}

All the colleges contacted responded to the survey giving a college response rate of $100 \%$. A total of 1662 students, were selected and invited to participate in the survey. Out of these 1596 students completed the questionnaires giving a response rate of $96 \%$. In six questionnaires the responses were incomplete and therefore excluded from analysis. The final analysis was carried out for 1590 questionnaires.

\section{Demographic characteristics}

The mean age of the students was 17.8 years (SD, 1.7). The median age was 17 years (minimum 14 years and maximum 32 years). The male to female ratio was 1.6:1. Out of 1590 students, 996 (62.6\%) were males and 594 (37.4\%) were females. A majority $(1383 / 1590,87 \%)$ of the students were Hindus, followed by Buddhists (157/ $1590,9.9 \%)$.

\section{Prevalence of tobacco use}

The prevalence of tobacco use according to gender is shown in Table 1. Overall prevalence of 'ever users' of tobacco products was $13.9 \%$. Prevalence among boys and girls was $20.5 \%$ and $2.9 \%$ respectively. Prevalence of 'current use' of tobacco products was $10.2 \%$ (cigarette smoking: $9.4 \%$, smokeless products: $6.5 \%$, both forms: $5.7 \%$ ). Majority of 'current users' of any tobacco product were 17 years or older. The prevalence of 'current users' increased after 17 years, the highest being among students who were 20 years or older (Figure 1). The differences in prevalence according to gender $(\mathrm{p}<0.001)$ and age $(\mathrm{p}<0.001)$ were statistically significant.

\section{Age at initiation of tobacco use}

Figure 2 shows age at initiation of tobacco use. Majority $(89.6 \%)$ of ever smokers had initiated smoking between the ages 12 and 18 years with a median age of 16 years. Among 'ever users' of chewable tobacco, 73.7\% had initiated between the ages 12 and 18 years with a median age of 15 years. Among ever smokers, 30.2\% had initiated before 15 years of age and $36.4 \%$ of 'ever users' of chewable tobacco had initiated before 15 years of age.

\section{Smoking pattern and access to tobacco products}

Among the current cigarette smokers, 58.7\% (88/150) were smoking at least one cigarette per day, 27.3\% (41/ $150)$ smoked once or twice a week and 14\% (21/150) smoked once or twice in a month. The average number of cigarettes smoked in a day was 3.5 (median 2, mode 1). About $62 \%$ of the students smoked one or two cigarettes per day. Most of them (66.7\%) smoked in tea stalls or restaurants followed by other public places (13.2\%). The others smoked at home (7.4\%) or a 'secret place' $(15.2 \%)$. Most $(67.8 \%)$ of them had purchased tobacco products from a shop or street vendor by themselves while $11.8 \%$ of the students purchased through a friend and $10.3 \%$ borrowed from friends. Six students also reported that they obtained tobacco products from a family member. Among the 104 current tobacco chewers, 34 (32.7\%) were using 'gutka' (mixture of tobacco and molasses), 27 (25\%) 'pan masala' (tobacco with aromatic spices) 24 (23.1\%) 'surti' (dried tobacco leaves for chewing), 12 (11.5\%) 'khaini' (tobacco with slaked lime and aromatic spices) and the remaining were using combinations of different types of chewable tobacco.

About 146 'current users' of tobacco products had responded for the average amount of money spent per day for buying tobacco products. They spent an average amount of 20 Nepalese rupees (NRS) [0.3 USD] per day (SD 23.6 NRS) ( 1 USD $\approx 66 \mathrm{NRS}$ ). The amount spent varied from 3 NRS [0.04 USD] to 130 NRS [1.96 USD] with

Table I: Prevalence of tobacco use (\%)

\begin{tabular}{llll}
\hline & Male* $(\mathbf{N}=\mathbf{9 9 6})$ & Female* $(\mathbf{N}=\mathbf{5 9 4})$ & Overall* (N = I590) \\
\hline Ever used any tobacco product & $20.5(17.9,22.9)$ & $2.9(1.5,4.2)$ & $13.9(12.2,15.6)$ \\
Currently smoking cigarettes & $14(11.8,16.1)$ & $1.9(0.8,2.9)$ & $9.4(8,10.9)$ \\
Currently using chewable tobacco & $9.3(7.5,11.1)$ & $1.9(0.8,2.9)$ & $6.5(5.3,7.8)$ \\
Currently using both forms of tobacco product & $8.2(6.5,9.9)$ & $1.5(0.5,2.5)$ & $5.7(4.6,6.9)$ \\
\hline
\end{tabular}

$* 95 \%$ confidence intervals 


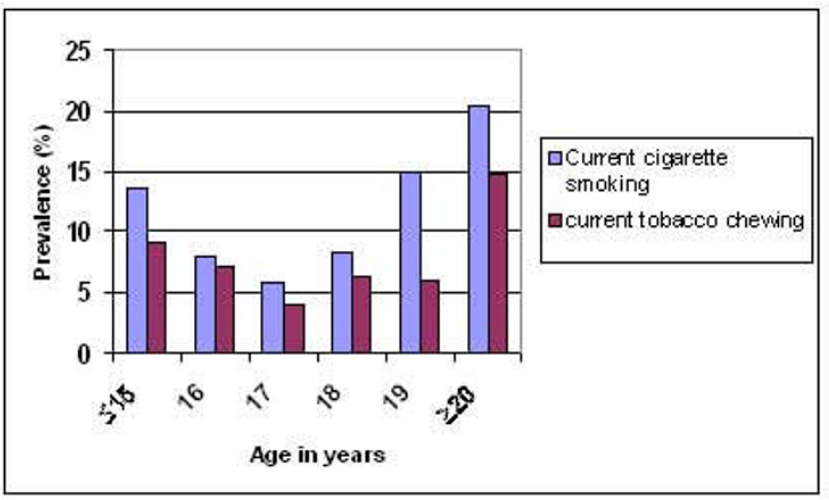

Figure I

Prevalence of current tobacco use according to age.

a median of 10 NRS [0.15 USD] (quartiles, 5 and 20). Out of 200 students who responded for the question "Do you have sufficient amount to buy tobacco products? 118 (59\%) had replied 'yes'.

\section{Media and advertising}

About $82 \%$ of the students had seen tobacco-related advertisements during the preceding 30 days of the survey. Magazines (59.7\%), newspapers (56.9\%), television $(48.7 \%)$, radio $(41.9 \%)$ and advertisement hoardings $(39.2 \%)$ were the media from which students were exposed to tobacco advertisements most often. Seven percent of the students had something (T-shirt, pen, bag etc) which had a cigarette brand logo on it. About 963 $(60.6 \%)$ students believed that cigarette advertisement or promotion can influence smoking use among students.

\section{Cessation}

The experiences of the current tobacco users of about cessation are shown in Table 2. A larger proportion of the students who were either currently smoking $(66.6 \%)$ or using chewable tobacco $(62.5 \%)$ thought that they can quit, whereas a lesser proportion $(63.3 \%$ and $57.7 \%$ respectively) had actually tried to quit. However, 55.3\% and $49.1 \%$ of the students who were currently smoking and chewing tobacco respectively had ever sought help to quit tobacco products. In most instances they sought advice from a friend or a girlfriend.

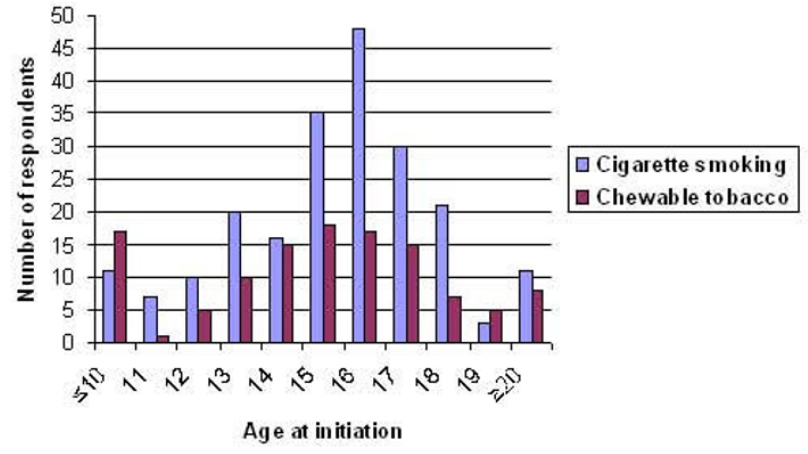

Figure 2

Age at initiation of tobacco use.

\section{Correlates of tobacco use}

The results of the multivariate logistic regression analysis are shown in Table 3. Among the demographic factors, being of older age and being a male were associated with tobacco use. Household asset score was used as a proxy for economic status. Students who had high asset score were more likely to be ever users of tobacco. Among predisposing factors knowledge about harm of tobacco use was protective for tobacco use. Among the enabling and reinforcing factors, tobacco use among the family members, friends and teachers were associated with tobacco use among the students. Students who had ever purchased tobacco products for their family members were more likely to have used any tobacco product.

\section{Discussion}

The dual purposes our study were to estimate the prevalence of tobacco use and assess the correlates. The sample size was determined using data from GYTS, Nepal and obtained a representative sample from the twin cities. We used a standardized survey instrument from GYTS [16] which made our data comparable to GYTS reports. However, our study was carried out among the college students instead of high school students in GYTS. In our study, the median age was 17 years which was higher than that of GYTS surveys. Despite this, the prevalence of ever tobacco use was slightly lesser than that reported by the GYTS, Nepal [10]. GYTS was carried out in central region which

Table 2: Experiences of the current tobacco users about cessation

\begin{tabular}{lll}
\hline Experience & Smokers $(\mathbf{N}=\mathbf{I 5 0})$ & Chewable tobacco (N = 104) \\
\cline { 2 - 3 } & Number of students (\%) & Number of students (\%) \\
\hline Had tried to quit & $95(63.3)$ & $60(57.7)$ \\
Thinks that he/she can quit & $100(66.6)$ & $65(62.5)$ \\
Sought help to quit & $83(55.3)$ & $51(49.1)$
\end{tabular}


Table 3: Multivariate analysis of determinants of tobacco use

\begin{tabular}{|c|c|c|c|c|}
\hline Variable & Number & $\begin{array}{l}\text { Adjusted } \\
\text { OR }\end{array}$ & $95 \%$ C I & p-value \\
\hline Age (Mean, SD) & $178,1.7$ & 1.13 & $1.04,1.22$ & 0.004 \\
\hline \multicolumn{5}{|l|}{ Gender } \\
\hline Female & 587 & I & & \\
\hline Male & 974 & 7.03 & $4.03,12.28$ & $<0.001$ \\
\hline \multicolumn{5}{|l|}{ Religion } \\
\hline Others & 206 & I & & \\
\hline Hindu & 1355 & 1.42 & $0.86,2.35$ & 0.162 \\
\hline \multicolumn{5}{|l|}{ Currently residence } \\
\hline Hostel/rented room & 679 & I & & \\
\hline With parents & 882 & 0.98 & $0.71,1.36$ & 0.93 \\
\hline \multicolumn{5}{|l|}{ Household asset score } \\
\hline Low & 1065 & I & & \\
\hline Medium & 394 & 1.71 & $0.95,3.07$ & 0.72 \\
\hline High & 102 & 1.52 & $1.06,2.12$ & 0.03 \\
\hline \multicolumn{5}{|c|}{ Are Tobacco products available at any place and anytime? } \\
\hline No/don't know & 679 & I & & \\
\hline Yes & 882 & 0.86 & $0.62,1.21$ & 0.38 \\
\hline \multicolumn{5}{|c|}{ Is the use of tobacco products banned in your college premises? } \\
\hline No/don't know & 624 & I & & \\
\hline Yes & 937 & 1.1 & $0.79,1.53$ & 0.59 \\
\hline \multicolumn{5}{|c|}{ Do you think that your teachers smoke or chew tobacco? } \\
\hline No/don't know & 499 & I & & \\
\hline Yes & 1109 & 1.93 & $1.38,2.71$ & $<0.001$ \\
\hline \multicolumn{5}{|l|}{ Do your close friends smoke or chew tobacco? } \\
\hline No/don't know & 871 & I & & \\
\hline Yes & 690 & 1.69 & $1.20,2.38$ & 0.003 \\
\hline Number family members who use tobacco & & 1.40 & $1.12,1.76$ & 0.003 \\
\hline \multicolumn{5}{|c|}{ Have you ever or currently buy tobacco products for your members? } \\
\hline No & 1109 & I & & \\
\hline Yes & 452 & 1.78 & $1.28,2.48$ & 0.001 \\
\hline Knowledge score (harms of tobacco use) & & 0.75 & $0.68,0.83$ & $<0.001$ \\
\hline \multicolumn{5}{|c|}{ In your opinion do tobacco-related advertisements influence tobacco consumption? } \\
\hline No/don't know & 610 & 1 & & \\
\hline Yes & 951 & 0.81 & $0.58,1.13$ & 0.214 \\
\hline
\end{tabular}

Only $156 \mathrm{I}$ cases were included in the final logistic regression due to missing data for some variables.

included both urban and rural areas but our study was carried out in urban area only. However, the prevalence of our study is lesser than that reported from Kerala, India [17] and USA where nearly one-third of the students were currently using tobacco products [18]. It is important to note the prevalence was low compared to western countries which have strict legislation, heavy taxes and massive information campaigns. Such difference was found in GYTS survey also [9]. Prevalence lower than that of western countries despite the lack of tobacco control measures may be explained by differences in socio-cultural milieu between these populations.

Globally, GYTS reports have shown that the difference among current smokers between boys and girls is smaller than that between men and women $[9,19]$. However, there was a yawning gap in prevalence between boys and girls in our study. A community-based survey from East- ern Nepal reported a similar rate of prevalence in the age group 14-25 years. However, the study reported that the prevalence of smoking increased with age [8]. Similar gender differentials and increasing trends of tobacco use with age was reported by WHO [20].

The study from Kerala, India has reported that the age at initiation appears to be declining [17]. The results of our study are similar to those reported from Kerala, India [17] and Indonesia [12]. The age at initiation for chewable products was lesser than that for smoking. This may be due to the growing popularity of the smokeless products accessible and available in small attractive sachets [21]. A good proportion $(5.7 \%)$ of students was currently using both forms of tobacco. It appears that smoking is often preceded by the use of chewable tobacco. The broad use of tobacco products among a substantial portion of this $16-20$ years age group could be an indication of a future 
increase in overall adult tobacco use. Less than half the students either tried to quit tobacco use or sought professional help to quit tobacco use. Therefore, counseling and quit-line programmes need to be started at the colleges for the benefit of the current users.

Most students $(78.7 \%)$ thought that street vendors, tea stalls and shops are selling tobacco products and $63.3 \%$ of the students responded that tobacco products are easily available. It has been suggested that banning the sales of tobacco products may be a deterrent to tobacco use among youth. However, there is no such legislation existing in Nepal [22]. Most students purchased tobacco products by themselves from street vendors or shops and were not denied by the virtue of their age. Average number of cigarettes smoked per day was about three. About three-fifths of the students smoked one or two cigarettes per day. One of the measures to control tobacco use is the ban of single stick sale. Such legislation also does not exist in Nepal [22]. The common place of smoking was tea stall or restaurants and public places. Legislations like restricted sales of tobacco products and ban of smoking in public places might be helpful in curbing the tobacco use among these young adults. A majority of the students had seen protobacco advertisements during the preceding 30 days from a wide range of media. However, this was not significantly different between ever tobacco users and never users. In Nepal there is no existing legislation about sampling and sponsoring by the tobacco industry [22]. There should be a complete ban of tobacco advertisements as partial ban has shown to be ineffective [23]. The WHO Framework Convention on Tobacco Control (FCTC) requires a comprehensive ban on advertising and promotion which may be ratified by the public health authorities in Nepal [24]. Measures like banning tobacco use in movies and television are thought to be sound public health policies as youth are influenced by movies and television [25].

The independent factors determining tobacco use among these college students were age, gender and household assets. Students from higher income group may be getting higher pocket money and therefore could afford to buy tobacco products. Among the other factors knowledge score was protective for tobacco use. Those students who had better knowledge of health risks of tobacco use were less likely to have 'ever used' any tobacco products. Similar observations were made in studies from Indonesia [12], and Argentina [26]. Therefore, it may be beneficial to introduce lessons on health risks of tobacco at schools and colleges.

Among the reinforcing factors the students who reported that one or more family members smoking or chewing tobacco were more likely to be ever users of tobacco. We investigated tobacco use among parents and siblings. As the number of family members using tobacco increased by a unit, the risk of tobacco used increased 1.5 times. Similarly having purchased tobacco products for a family member was also associated with tobacco use. The students who had friends who used tobacco products and who reported that their teachers use tobacco products were also likely to use tobacco products. Similar results have been reported by studies from Indonesia [12], Argentina [26] and Kerala, India [17]. Even school children have opined that parents [27] and peers have strong influence on tobacco use amongst youth [28]. However, there is a possibility that tobacco users may have reported about users in their environment due to selective perception.

Nearly $40 \%$ reported that at least one family member uses tobacco. Despite schools and colleges of Nepal being tobacco-free, the teachers' tobacco use behavior may have influenced students. Tobacco use among the family members, in particular parents and siblings may also have an influence. Similarly in the college environment peer pressure also appears to play a role for adopting tobacco use as a way of life. Tobacco use among adults, lack of ban on smoking in public places, exposure to tobacco use at home may have created an opinion that tobacco use is a 'social norm'. Behavior change communication may not be successful unless adults change their behavior. Despite good knowledge about health risks of tobacco use among all the students, their perception of individual risk may be poor. Educational efforts should focus on dispelling the misconceptions created in the young minds by the media.

\section{Limitations}

Our study had some limitations. Our survey was cross-sectional, and smoking status was by self-report. Therefore, some students may have under reported their tobacco use. Moreover the existing taboo about tobacco use, some female students in particular might also be underreported. Some students who were using tobacco might have been absent from the college on the day of survey. We did not have an opportunity to interview them. The study was carried out in colleges of two urban locations only. So our results cannot be extrapolated to the rest of Nepal.

\section{Conclusion}

Tobacco use in any form (smoking or smokeless) is prevalent among the college students. Cigarette smoking was the most popular form of tobacco use. Older male college students were more likely to use tobacco. Knowledge of health risk, household asset score, peer influences and social norms like tobacco use among teachers and family members, buying tobacco products for a family member were associated with tobacco use. Targeted school/college-based intervention strategies by counseling and education are necessary. Enforcement of regulations on sale and advertisements of tobacco products may also be useful. Legislations on use of tobacco products need to be enforced to decrease availability, accessibility and afford- 
ability of tobacco products. Policies to bring about changes in acceptability of tobacco use (social norms) among parents, teachers may also help to curb the tobacco use among college students.

\section{Competing interests}

The author(s) declare that they have no competing interests.

\section{Authors' contributions}

CTScontributed to the design and protocol of the study, participated in the data collection, was the primary researcher and drafted the manuscript for publication. PVKhelped with the design of the study, data analysis, development of questionnaire and assisted in preparation of first draft. JPDesigned and conducted the data analysis, assisted in manuscript preparation and criticized the earlier drafts of the manuscript. RGMconceived the study, set up the design, development of questionnaire and criticized the earlier drafts of the manuscript. All authors read and approved the final manuscript for submission for publication.

\section{Acknowledgements}

The authors are grateful to all the principals and teachers of the colleges for their kind cooperation and assistance to carry out this survey. The authors and express their gratitude to all the students who participated in this survey.

\section{References}

I. Ezzati M, Lopez AD, Rodgers A, Vander Hoorn S, Murray CJL, the Comparative Risk Assessment Collaborating Group: Selected major risk factors and global and regional burden of disease. Lancet 2002, 360: 1347-60.

2. Peto R, Lopez AD, Boreham J, Thun M, Heath C Jr: Mortality from smoking in developed countries 1950-2000: indirect estimation from National Vital Statistics. Oxford: Oxford University Press; 1994.

3. World Health Organization: The impact of chronic diseases in Nepal. WHO 2002 [http://www.who.int/chp/ chronic disease report/en/]. (Accessed on January 2, 2007)

4. WHO South-East Asia Region: WHO World Health Survey. WHO Global InfoBase Version: I.292beta 200I.

5. Pandey MR, Neupane RP, Gautam A: Epidemiological study of tobacco smoking behaviour among adults in a rural community of the hill region of Nepal with special reference to attitude and beliefs. Int J Epidemiol 1988, I 7:535-4I.

6. Pandey MR, Venkatramaiah SR, Neupane RP, Gautam A: Epidemiological study of tobacco smoking behaviour among young people in a rural community of the hill region of Nepal with special reference to attitude and beliefs. Community Med 1987, 9: I 10-20.

7. Jha N, Upadhyay MP, Lakhey S, Yadav BK, Baral DD, Ghartichhetri PS: Smoking and smokers in Sunsari, Nepal. J Nep Med Assoc 1999, 38:7-13.

8. Niraula SR: Tobacco use among women in Dharan, eastern Nepal. J Health Popul Nutr 2004, 22:68-74.

9. Warren CW, Jones NR, Eriksen MP, Asma S, Global Tobacco Surveillance System (GTSS) collaborative group: Patterns of global tobacco use in young people and implications for future chronic disease burden in adults. Lancet 2006, 367:749-53.

10. Pandey MR, Pathak RP: Challenges of tobacco use behavior in central development region of Nepal: Global Youth Tobacco Survey Collaborative Group. Nepal GYTS Fact Sheet 2002 [http:/ /www.cdc.gov/tobacco/global/GYTS/reports/searo/200I/ nepal report.htm]. (Accessed on January 2, 2007)
II. Nichter M, Nichter M, Van Sickle D: Popular perceptions of tobacco products and patterns of use among male college students in India. Soc Sci Med 2004, 59:4I 5-3I.

12. Martini S, Sulistyowati M: The Determinants of Smoking Behavior among Teenagers in East Java Province, Indonesia. Health, Nutrition and Population (HNP) Discussion Paper. Economics Of Tobacco Control Paper No. 32. World Bank 2003 [http://sitere sources.worldbank.org/HEALTHNUTRITIONANDPOPULATIOResources/281627-1095698/40167/IndonesiaYouthSmokingFi nal.pdf].

13. United Nations (UN): Human Development Report. Geneva: UN; 2002.

14. Central Bureau of Statistics, National Planning Commission Secretariat: Population Census 200I. Nepal: His Majesty Government; 2001.

15. Green L, Kreuter M: An Educational and Environmental Approach. In Health Promotion Planning 2 nd edition. Mayfield Publishing Company, Toronto; 1991.

16. Center for Disease Control and Prevention: Global Youth Tobacco Survey. [http://www.cdc.gov/tobacco/global/GYTS/ intro.htm]. Accessed on Nov. 30, 2006

17. Pradeepkumar AS, Mohan S, Gopalakrishnan P, Sarma PS, Thankappan $K R$, Nichter $M$ : Tobacco use in Kerala: findings from three recent studies. Natl Med J India 2005, I 8: |48-53.

18. Rigotti NA, Lee JE, Wechsler $H$ : US college students' use of tobacco products: results of a national survey. JAMA 2000, 284:699-705.

19. WHO: Tobacco or health: a global status report. Geneva: World Health Organization; 1997.

20. Pande B, Karki Y, Plant K: A study on tobacco economics in Nepal. In The SuRF Report I. Surveillance of Risk Factors related to Noncommunicable Diseases: Current status of global data Edited by: Strong K, Bonita R. Geneva: WHO; 200I. 2003

21. Shimkhada R, Peabody JW: Tobacco control in India. Bull World Health Organ 2003, $81: 48-51$.

22. World Bank. South-east Asia region: $\mathbf{N}$ epal smoking prevalence tobacco economy. World Bank 200I [http://siteresources.world bank.org/INTETC/Resources/375990-III2106769274/Nepal.pdf].

23. Jha P, Chaloupka FJ: Curbing the epidemic: Governments and the economics of tobacco control. Washington DC: The World Bank; 1999.

24. World Health Organization: Updated status of the WHO framework convention on tobacco control. [http://www.who.int/ tobacco/framework/countrylist/en/index.html]. (accessed June 8, 2007)

25. Reddy KS, Arora M: Ban on tobacco use in films and television represents sound public health policy. Natl Med J India 2005, I8:115-8.

26. Morello P, Duggan A, Adger $\mathrm{H}$ Jr, Anthony JC, Joffe A: Tobacco use among high school students in Buenos Aires, Argentina. Am J Public Health 2001, 91:219-24.

27. Staten RR, Noland M, Rayens MK, Hahn E, Dignan M, Ridner SL: Social influences on cigarette initiation among college students. Am J Health Behav 2007, 3 I:353-62.

28. Mishra A, Arora M, Stigler MH, Komro KA, Lytle LA, Reddy KS, Perry $C L$ : Indian youth speak about tobacco: results of focus group discussions with school students. Health Educ Behav 2005, 32:363-79.

\section{Pre-publication history}

The pre-publication history for this paper can be accessed here:

http://www.biomedcentral.com/1471-2458/8/97/prepub 